\title{
Supplementary Material to: Specification of informative prior distributions for multinomial models using vine copulas
}

\author{
Kevin J. Wilson
}

July 21, 2017

\section{Vines}

A vine (vine copula, pair copula construction) on $n$ variables [1] is a nested set of trees, where the edges of tree $j$ are the nodes of tree $j+1$ (for $j=1, \ldots, n-2$ ), and each tree has the maximum number of edges. A regular vine on $n$ variables is a vine in which two edges in tree $j$ are joined by an edge in tree $j+1$ only if these edges share a common node, for $j=1, \ldots, n-2$. There are $n(n-1) / 2$ edges in a regular vine on $n$ variables. The definition is given below.

Definition $1 \mathcal{V}$ is a regular vine on $n$ elements if

1. $\mathcal{V}=\left(T_{1}, \ldots, T_{n-1}\right)$.

2. $T_{1}$ is a connected tree with nodes $N_{1}=\{1, \ldots, n\}$ and edges $E_{1}$; for $i=$ $2, \ldots, n-1, T_{i}$ is a connected tree with nodes $N_{i}=E_{i-1}$.

3. For $i=2, \ldots, n-1$, if $a$ and $b$ are nodes of $T_{i}$ connected by an edge in $T_{i}$, where $a=\left\{a_{1}, a_{2}\right\}, b=\left\{b_{1}, b_{2}\right\}$, then exactly one of the $a_{i}$ equals one of the $b_{i}$.

We can use a vine to construct a vine distribution on $n$ variables. This vine distribution will become our prior distribution. Each node in the first tree of the vine represents an unconditional variable and in each subsequent tree each node represents a conditional variable. The edges represent dependency between the adjacent nodes in the form of unconditional and conditional bivariate copulas. A bivariate copula is a distribution function on $[0,1]^{2}$ such that, for variables $\left(X_{1}, X_{2}\right)$ with continuous probability density functions (PDFs) $f_{i}\left(x_{i}\right)$ and cumulative distribution functions (CDFs) $F_{i}\left(x_{i}\right), F\left(x_{1}, x_{2}\right)=C\left(F_{1}\left(x_{1}\right), F_{2}\left(x_{2}\right)\right)$ and $f\left(x_{1}, x_{2}\right)=f_{1}\left(x_{1}\right) f_{2}\left(x_{2}\right) c\left(F_{1}\left(X_{1}\right), F_{2}\left(X_{2}\right)\right)$, where $C(\cdot, \cdot)$ and $c(\cdot, \cdot)$ are the copula CDF and PDF respectively. Thus we can use a bivariate copula to specify the distribution of $X_{1}, X_{2}$ by first specifying the marginal distributions $f_{1}\left(x_{1}\right), f_{2}\left(x_{2}\right)$ and then separately specifying the dependence via the copula $C\left(F_{1}\left(x_{1}\right), F_{2}\left(x_{2}\right)\right)$.

We can represent a vine distribution as in the following [2]. 
Theorem 1 Let $\mathcal{V}=\left(T_{1}, \ldots, T_{n-1}\right)$ be a regular vine on $n$ elements. For each edge $e(j, k) \in T_{i}, i=1, \ldots, n-1$, let the conditional copula and copula density of $\left(X_{j}, X_{k}\right)$ conditioned on variables in the set $D_{e}$ be $C_{j, k \mid D_{e}}$ and $c_{j, k \mid D_{e}}$ respectively. Let the marginal distributions $F_{i}$ with densities $f_{i}, i=1, \ldots, n$ be given. Then the vine distribution is uniquely determined and has a density given by

$$
f_{1, \ldots, n}\left(x_{1}, \ldots, x_{n}\right)=\prod_{i=1}^{n} f_{i}\left(x_{i}\right) \prod_{j=1}^{n-1} \prod_{e(j, k) \in E_{i}} c_{j, k \mid D_{e}}\left(F_{j \mid D_{e}}\left(x_{j}\right), F_{k \mid D_{e}}\left(x_{k}\right)\right) .
$$

There are different classes of regular vine, two of the most widely used being canonical vines (C-vines) and drawable vines (D-vines). For our purposes, we will take advantage of $\mathrm{D}$-vines, which are easy to visualise. The general structure of a D-vine in 5 dimensions is given in Figure 1.

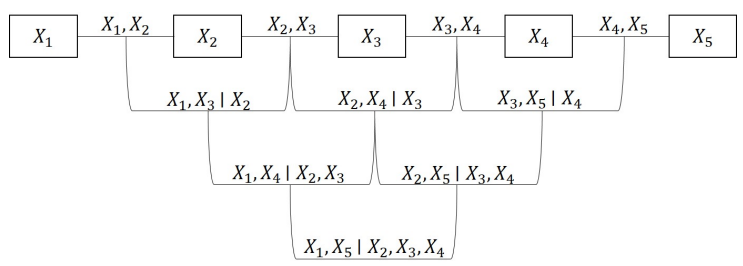

Figure 1: The structure of a general D-vine in 5 dimensions.

We see each of the 4 trees in the vine and the bivariate copulas to be specified in each. For example, in the third tree of the vine we see that we need to specify conditional copulas between $X_{1}, X_{4}$ conditional on the values of $X_{2}, X_{3}$ and $X_{2}, X_{5}$ conditional on $X_{3}, X_{4}$. In all, there are 10 bivariate copulas needed to fully specify the vine.

We can use Theorem 1 to write down the joint density of $\boldsymbol{X}=\left(X_{1}, X_{2}, X_{3}, X_{4}, X_{5}\right)$ using this D-vine. It is

$$
\begin{aligned}
f_{1,2,3,4,5}(\boldsymbol{x})= & f_{1}\left(x_{1}\right) f_{2}\left(x_{2}\right) f_{3}\left(x_{3}\right) f_{4}\left(x_{4}\right) f_{5}\left(x_{5}\right) \\
& \times c_{1,2}\left(F_{1}\left(x_{1}\right), F_{2}\left(x_{2}\right)\right) c_{2,3}\left(F_{2}\left(x_{2}\right), F_{3}\left(x_{3}\right)\right) c_{3,4}\left(F_{3}\left(x_{3}\right), F_{4}\left(x_{4}\right)\right) c_{4,5}\left(F_{4}\left(x_{4}\right), F_{5}\left(x_{5}\right)\right) \\
& \times c_{1,3 \mid 2}\left(F_{1 \mid 2}\left(x_{1} \mid x_{2}\right), F_{3 \mid 2}\left(x_{3} \mid x_{2}\right)\right) c_{2,4 \mid 3}\left(F_{2 \mid 3}\left(x_{2} \mid x_{3}\right), F_{4 \mid 3}\left(x_{4} \mid x_{3}\right)\right) \\
& \times c_{3,5 \mid 4}\left(F_{3 \mid 4}\left(x_{3} \mid x_{4}\right), F_{5 \mid 4}\left(x_{5} \mid x_{4}\right)\right) \\
& \times c_{1,4 \mid 2,3}\left(F_{1 \mid 2,3}\left(x_{1} \mid x_{2}, x_{3}\right), F_{4 \mid 2,3}\left(x_{4} \mid x_{2}, x_{3}\right)\right) c_{2,5 \mid 3,4}\left(F_{2 \mid 3,4}\left(x_{2} \mid x_{3}, x_{4}\right), F_{5 \mid 3,4}\left(x_{5} \mid x_{3}, x_{4}\right)\right) \\
& \times c_{1,5 \mid 2,3,4}\left(F_{1 \mid 2,3,4}\left(x_{1} \mid x_{2}, x_{3}, x_{4}\right), F_{5 \mid 2,3,4}\left(x_{5} \mid x_{2}, x_{3}, x_{4}\right)\right)
\end{aligned}
$$

A D-vine on $n$ variables has its structure uniquely defined by the ordering of the variables in the first tree.

In the above derivation each of the conditional copulas depend explicitly on the variables they are conditioned on. If we relax this condition, and assume that the conditioning affects only the conditional distribution of the variables, e.g., $F_{i \mid j}\left(x_{i} \mid x_{j}\right)$, then we can utilise the idea of a simplified vine. 
Definition 2 A simplified vine is a vine in which all conditional bivariate copulas do not depend on the variables that are conditioned on.

[3] show that this assumption is often reasonable and, even when not satisfied, can provide reasonable approximation. For a more comprehensive explanation of vines see Chapter 4 of [4].

\subsection{Bivariate copulas}

There are many parametric bivariate copulas in the literature. Two important families are inversion copulas, which include the Gaussian and t-copulas, and Archimedian copulas, which include the Clayton, Gumbel and Frank copulas. A bivariate copula in the inversion family takes the form

$$
C\left(F_{1}^{(0)}\left(\tilde{\theta}_{1}\right), F_{2}^{(0)}\left(\tilde{\theta}_{2}\right)\right)=G_{1,2}\left[G_{1}^{-1}\left(F_{1}^{(0)}\left(\tilde{\theta}_{1}\right)\right), G_{2}^{-1}\left(F_{2}^{(0)}\left(\tilde{\theta}_{2}\right)\right)\right],
$$

where $G_{i}(\cdot)$ and $G_{1,2}(\cdot, \cdot)$ are the marginal and joint distributions of the copula (e.g. t-distributions). A bivariate copula in the Archimedian family takes the form

$$
C\left(F_{1}^{(0)}\left(\tilde{\theta}_{1}\right), F_{2}^{(0)}\left(\tilde{\theta}_{2}\right)\right)=\phi^{[-1]}\left[\phi\left(F_{1}^{(0)}\left(\tilde{\theta}_{1}\right)\right)+\phi\left(F_{2}^{(0)}\left(\tilde{\theta}_{2}\right)\right)\right]
$$

where $\phi(\cdot)$ is a strictly decreasing convex function with $\phi(1)=0$ and $\phi^{[-1]}(u)$ is the inverse of $\phi(u)$ for $0 \leq u \leq \phi(0)$ and zero otherwise.

Kendall's tau is a suitable measure of correlation within a copula. For bivariate copulas, Kendall's Tau can be calculated explicitly [5] using

$$
\tau_{i, j}=4 \int_{0}^{1} \int_{0}^{1} C\left(F_{i}^{(0)}\left(\tilde{\theta}_{i}\right), F_{j}^{(0)}\left(\tilde{\theta}_{j}\right)\right) d C\left(F_{i}^{(0)}\left(\tilde{\theta}_{i}\right), F_{j}^{(0)}\left(\tilde{\theta}_{j}\right)\right)-1 .
$$

In Table 1 we provide summaries of some commonly used bivariate copulas. For a more comprehensive list of copulas and their properties see [5]. In each case we give the CDF for $u=F_{i}^{(0)}\left(\tilde{\theta}_{i}\right), v=F_{j}^{(0)}\left(\tilde{\theta}_{j}\right)$, where $i \neq j$, the set of possible values for the copula parameters and the range of possible values of Kendall's tau for the copula.

In the table, $\Phi_{\lambda}$ is the CDF of a standard bivariate Normal distribution with correlation $\lambda, \Phi$ is the CDF of a standard univariate Normal distribution and $t_{\lambda_{2}}$ is the CDF of a t-distribution with $\lambda_{2}$ degrees of freedom.

We see that the Normal, t- and Frank copulas are sufficiently flexible to allow specification of the entire range of Kendall correlations between -1 and 1 . The Gumbel and Clayton copulas are suitable for positive values of the Kendall correlation and their rotated versions are suitable for negative values.

\section{References}

[1] T. Bedford and R. Cooke. Vines - a new graphical model for dependent random variables. Annals of Statistics, 30:1031-1068, 2002. 
[2] T. Bedford, A. Daneshkhah, and K. Wilson. Approximate uncertainty modeling in risk analysis with vine copulas. Risk Analysis, 2016.

[3] I. Haff, K. Aas, and A. Frigessi. On the simplified pair-copula construction simply useful or too simplistic? Journal of Multivariate Analysis, 101:12961310, 2010.

[4] D. Kurowicka and R. Cooke. Uncertainty Analysis with High Dimensional Dependence Modelling. Wiley, 2006.

[5] R. Nelson. An Introduction to Copulas. Springer, 2006. 


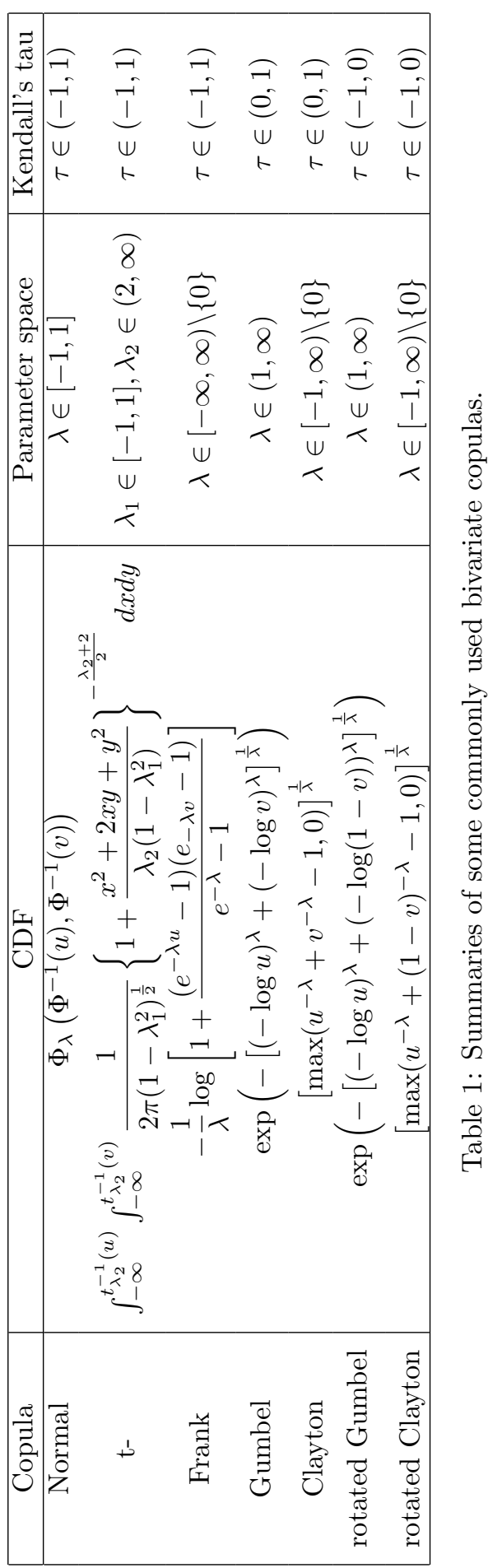

